From ballistic transport to tunneling in electromigrated ferromagnetic breakjunctions.

Bolotin, Kirill I; Kuemmeth, Ferdinand; Pasupathy, Abhay N; Ralph, D C

Published in:

Nano Letters

DOI:

$10.1021 / \mathrm{nl} 0522936$

Publication date:

2006

Document version

Publisher's PDF, also known as Version of record

Citation for published version (APA):

Bolotin, K. I., Kuemmeth, F., Pasupathy, A. N., \& Ralph, D. C. (2006). From ballistic transport to tunneling in electromigrated ferromagnetic breakjunctions. Nano Letters, 6(1), 123-127. https://doi.org/10.1021/nl0522936 


\title{
From Ballistic Transport to Tunneling in Electromigrated Ferromagnetic Breakjunctions
}

\author{
Kirill I. Bolotin, F. Kuemmeth, Abhay N. Pasupathy, and D. C. Ralph \\ Laboratory of Atomic and Solid State Physics, Cornell University, \\ Ithaca, New York 14853
}

Received November 20, 2005

\begin{abstract}
We fabricate ferromagnetic nanowires with constrictions whose cross section can be reduced gradually from $100 \times 30 \mathrm{~nm}^{2}$ to the atomic scale and eventually to the tunneling regime by means of electromigration. The contacts are mechanically and thermally stable. We measure lowtemperature magnetoresistances (MR) $<3 \%$ for contacts $<400 \Omega$, reproducible MR variations that are nonmonotonic in the regime $400 \Omega$ $25 \mathrm{k} \Omega$, and a maximum MR of $80 \%$ for atomic-scale widths. These results for devices $>400 \Omega$ differ from previous room-temperature studies of electrodeposited devices. For samples in the tunneling regime, we observe large fluctuations in MR, between -10 and $85 \%$.
\end{abstract}

The entry of a magnetic domain wall into a nanometer-scale magnetic contact can cause magnetoresistance $(M R=[R(\mathrm{AP})$ - $R(\mathrm{P})] / R(\mathrm{P})$, where $R(\mathrm{AP})$ is the resistance with an antiparallel orientation for the magnetizations in the electrodes and $R(\mathrm{P})$ is the resistance with parallel magnetizations). Several different interesting mechanisms can contribute, depending on the size of the contact. If the device diameter is greater than tens of nanometers, then the largest contribution to the domain wall resistance is generally the anisotropic MR, a difference in the resistivity of a magnetic material depending on whether the magnetic moment is oriented parallel or perpendicular to the current. This contribution is relatively small, typically giving maximum MR values of a few percent. ${ }^{1}$ As the contact diameter is reduced, the width of the domain wall can be constrained by the geometry and decreases in proportion to the contact width. ${ }^{2}$ Eventually a new mechanism of MR may become dominant if a domain wall is sufficiently narrow that the spin of a conduction electron cannot follow the direction of the local magnetization adiabatically. ${ }^{3}$ In that case, the domain wall can produce increased electron scattering that is analogous to the giant magnetoresistance effect in magnetic multilayers. ${ }^{4}$ For very small metallic contacts, approaching the single-atom diameter regime, room-temperature values of MR as large as $200 \%$ to $100000 \%$ have been reported ${ }^{5-7}$ and ascribed to a "ballistic magnetoresistance" effect involving scattering of electrons from an atomically abrupt domain wall. However, these large effects have not been reproducible in other device geometries, ${ }^{8-11}$ and there is now considerable evidence that magnetostriction and magnetostatic forces can

\footnotetext{
* Corresponding author. E-mail: ralph@ccmr.cornell.edu.
}

produce artifacts in similar experiments by causing the physical structure of the contact to change as the magnetic field is varied.9,12 Finally, if the contact diameter is reduced beyond the single-atom limit, then it enters the tunneling regime. MR in that regime reflects the spin polarization of tunneling electrons, and for a nanoscale device it is interesting to ask to what extent the MR depends on the atomicscale geometry of the contacts, rather than simply the spin polarization of the bulk electron density of states. ${ }^{13,14}$

To avoid artifacts of mechanical instabilities and achieve reliable measurements of the intrinsic magnetoresistance of atomic-scale magnetic contacts, we argue that at least three conditions should be met: (i) the magnetic electrodes should be attached rigidly to a nonmagnetic substrate with no suspended parts so that influences of magnetostriction and magnetostatic forces are minimized, (ii) the measurements should be performed at cryogenic temperatures so that the contact region is thermally stable (even at a fixed magnetic field, room temperature contacts are typically unstable on time scales of seconds ${ }^{10}$ ), and (iii) the geometry of the magnetic electrodes should be designed so that their moments can be controlled between reliably antiparallel and parallel configurations. Although refs 11 and 15 fulfilled these conditions for larger devices, the experiments we report here are the first to fulfill all three conditions for contact sizes down to the atomic scale. We fabricate samples in which two thin-film ferromagnets are connected by a small magnetic constriction that can be controllably narrowed by electromigration from about $100 \times 30 \mathrm{~nm}^{2}$ to the atomic scale and finally to a tunnel junction. This allows us to study the low-temperature MR as the contact region between the 


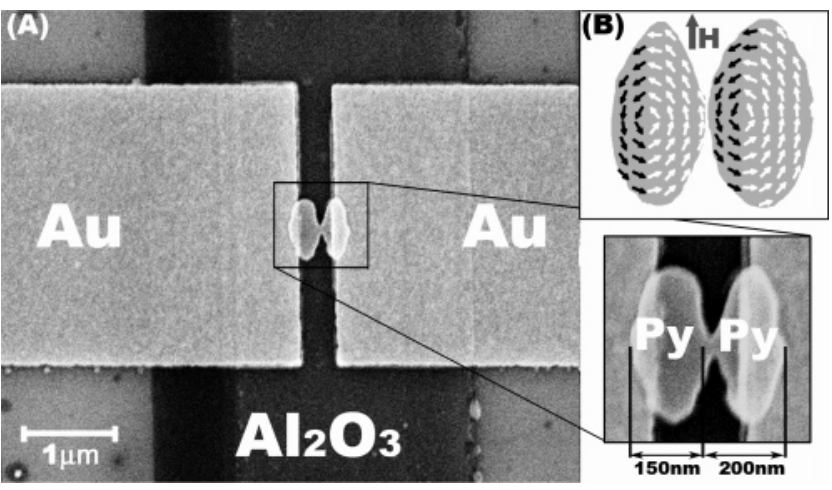

Figure 1. (A) Scanning electron micrograph of a finished device. Gold electrodes are used to contact two permalloy thin-film magnets (inset) on top of an oxidized aluminum gate. The irregular shape of the Py electrodes results from imperfect liftoff during fabrication. (B) Micromagnetic modeling showing antiparallel magnetic alignment across the tunneling gap in an applied magnetic field of $H=$ $66 \mathrm{mT}$.

two ferromagnets is progressively narrowed in a single sample. Our results for MR versus contact size are consistent with previous experiments for large contacts $(<200 \Omega)$, but we observe previously unreported systematic changes in a regime of intermediate resistance $(400 \Omega-25 \mathrm{k} \Omega)$ and also the tunneling regime $(>25 \mathrm{k} \Omega)$ that can be compared quantitatively to theoretical predictions. Our results differ significantly from previous experiments that were conducted at room temperature or that employed device geometries that were more sensitive to magnetostriction and magnetostatic forces. ${ }^{5-9,12}$

Our device design builds on the approach taken by Pasupathy et al., ${ }^{16}$ in which the angle between the moments in the two magnetic electrodes could be manipulated by fabricating them with different shapes so that they undergo magnetic reversal at different values of the applied magnetic field. However, the shapes of the electrodes used in ref 16 were not optimal in that an accurate antiparallel (AP) configuration of the two moments could not be obtained reliably. In the design used here (shown in Figure 1), the electrodes are elongated along the axis perpendicular to the constriction that connects them. A magnetic field is applied parallel to the long axis of the electrodes. From simple magnetostatic considerations, one expects that dipole interactions between the two electrodes will favor AP alignment with a domain wall in the constriction region for small applied magnetic fields. For sufficiently strong applied fields, both moments align parallel $(\mathrm{P})$ to the field. We have performed micromagnetic modeling of this geometry using the OOMMF code. ${ }^{17} \mathrm{We}$ find that the electrodes can access some states other than the simple uniform P and AP states at intermediate values of field (see the vortex states in Figure 1B), but the local magnetizations on opposite sides of the constriction region still accurately remain either $\mathrm{P}$ or $\mathrm{AP}$. When the electrode magnetizations are AP and they are connected by a narrow bridge of ferromagnetic metal, a domain wall forms inside the bridge. We expect that the rotation of the magnetization within the domain wall occurs in plane of the thin-film electrodes because the demagnetiza-

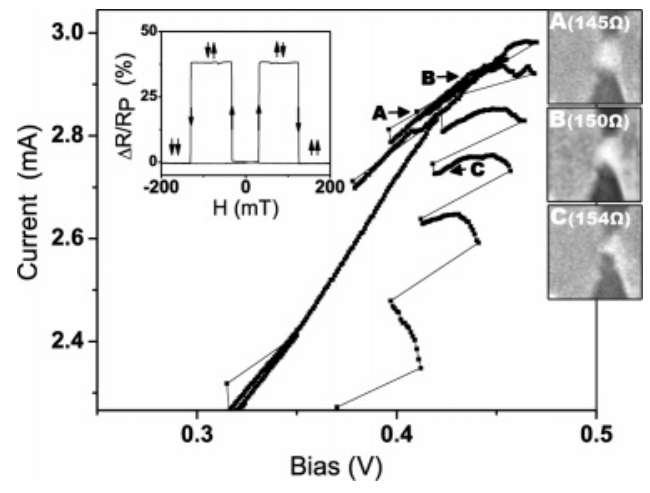

Figure 2. The cross section of a constriction is reduced in stages $(\mathrm{A}-\mathrm{C})$ by ramping the bias voltage repeatedly until electromigration begins and then quickly decreasing the bias, following a procedure similar to that in ref 19 . The SEM micrographs illustrate the gradual narrowing of the constriction, which appears bright in these images. Inset: resistance as a function of magnetic field for a tunneling device exhibiting abrupt switching between parallel and antiparallel magnetic states.

tion field of the film will prevent the formation of a Bloch wall. However, the detailed structure of the domain wall is expected to depend on the atomic arrangement in the bridge connecting the two ferromagnets. ${ }^{14}$

We fabricate the devices on top of an oxidized aluminum gate electrode (not used in this experiment), on a silicon substrate by using aligned steps of electron-beam lithography $^{18}$ and thermal evaporation to first deposit gold contact pads 20-nm-thick and then the magnetic permalloy electrodes 30-nm-thick, with a 100-nm-wide permalloy bridge connecting the magnetic electrodes (Figure 1A). We chose permalloy for its low crystalline anisotropy, low magnetostriction, and high spin polarization at the Fermi level. To vary the size of the bridge connecting the two magnetic electrodes, we use controlled electromigration ${ }^{19}$ at liquid helium temperatures. We slowly ramp the voltage across the constriction while monitoring the current. At roughly $3 \mathrm{~mA}$ $\left(10^{8} \mathrm{~A} / \mathrm{cm}^{2}\right)$, electromigration begins (as indicated by an increase in differential resistance), at which point the acquisition software quickly lowers the bias. Repeating this procedure allows us to increase the resistance of the junction to any desired value between $100 \Omega$ and $1 \mathrm{k} \Omega$ with better than $10 \%$ accuracy and to values between $1 \mathrm{k} \Omega$ and $20 \mathrm{k} \Omega$ with better than $50 \%$ accuracy. Once electromigration is completed, the junctions are mechanically stable as long as they remain at low temperature, as indicated by resistances that remain unchanged within measurement accuracy on the time scale of days.

We have imaged the process of controlled electromigration inside a scanning electron microscope using test samples at room temperature. Figure $2 \mathrm{~A}-\mathrm{C}$ shows the gradual narrowing of the constriction as electromigration proceeds, and demonstrates that electromigration produces a single break near the narrow region of the permalloy bridge. Once the permalloy constriction becomes narrower than about $10 \mathrm{~nm}$, its structure cannot be resolved in the SEM. Devices for which electromigration is allowed to proceed to form a tunneling gap exhibit magnetic tunnel-junction characteristics 
(Figure 2, inset), with stable switching between well-defined $\mathrm{P}$ and AP states. This agrees well with the micromagnetic simulations. Conductance versus voltage measurements in the tunneling regime at $4.2 \mathrm{~K}$ (discussed below) show no Coulomb-blockade or Kondo behavior, demonstrating that electron transport occurs via simple tunnel junctions rather than any electromigration-formed nanoparticles. ${ }^{20-21}$

We perform transport measurements at $4.2 \mathrm{~K}$ with the samples either immersed in liquid helium or in cryogenic vacuum to achieve the stability provided by low temperatures and to minimize the possibility of oxidation during and after the electromigration process. Initially, the resistance of each 100 -nm-wide device is approximately $60 \Omega$. The constriction between magnetic electrodes is then progressively narrowed by electromigration, with magnetoresistance measurements made after each stage. When the resistance begins to approach a significant fraction of $h / e^{2}=25.8 \mathrm{k} \Omega$, the transport is likely to be dominated by ballistic transport through just a few apex atoms. Finally, for devices with resistances of more than approximately $h / e^{2}$ the transport is dominated by electron tunneling. We find that the MR properties of the devices are qualitatively different in the regimes of low resistance $(<400 \Omega)$, intermediate resistance (400 $\Omega-25 \mathrm{k} \Omega$ ), and tunneling $(>25 \mathrm{k} \Omega$ ), so we will analyze these regimes separately below.

When the resistance of a device is low $(<400 \Omega)$ it increases smoothly as electromigration proceeds. The crosssection of the constriction varies from $100 \times 30 \mathrm{~nm}^{2}(60$ $\Omega$ ) to approximately $1 \mathrm{~nm}^{2}(400 \Omega)$, with the latter estimate based on the Sharvin formula. ${ }^{22}$ In this regime we find small $(<3 \%)$ positive MR, which increases as the constriction is narrowed (Figure 3A). This is consistent with other recent experiments ${ }^{15,23}$ and described well by the semiclassical theory of Levy and Zhang. ${ }^{3}$ In this theory, the resistance of the domain wall scales inversely with its width and the MR typically ranges from $0.7 \%$ to $3 \%$ for bulk ferromagnets.

The resistance range from $400 \Omega$ to $25 \mathrm{k} \Omega$ corresponds to a crossover between ballistic transport through just a few atoms and tunneling. In this regime, the resistance of the device increases in discrete steps during electromigration and the process is less controllable. Similar behavior is seen in conventional mechanical break junctions and corresponds to the rearrangement of atoms in the constriction. ${ }^{24}$ In this intermediate regime, the value of MR exhibits pronounced dependence on the resistance of the device. The MR has a minimum for resistances above $1 \mathrm{k} \Omega$, and typically changes sign here to give negative values. Negative MR in nanoscale contacts has been predicted previously. ${ }^{14,25}$ As the resistance is increased further into the $\mathrm{k} \Omega$ range, our measured MR increases gradually to positive values of $10-40 \%$. These trends are reproducible, although the exact dependence of the MR on resistance differs from device to device. The highest MR that we have observed was $80 \%$ for a device with a resistance of $14.5 \mathrm{k} \Omega$. The MR values that we measure in the point contact regime are smaller than expected from scaling results of the semiclassical theory. ${ }^{3}$ This difference is not surprising when the current is transmitted through just a few quantum channels. ${ }^{14}$

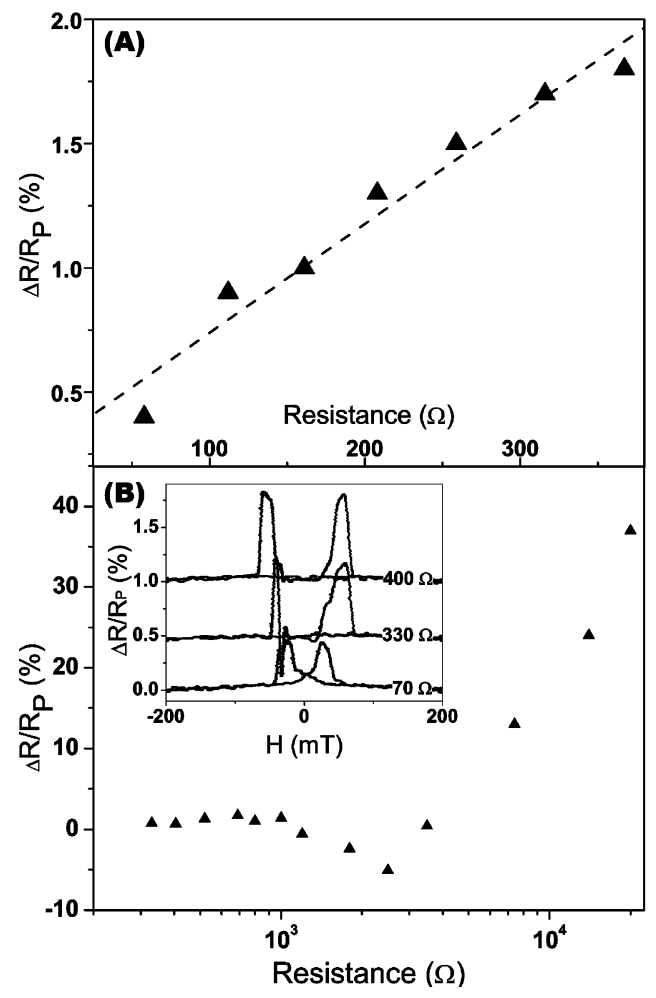

Figure 3. (A) Magnetoresistance as a function of resistance in the range less than $400 \Omega$ (device I). (B) Magnetoresistance as a function of resistance in the range $60 \Omega-15 \mathrm{k} \Omega$ (device II). Inset: switching behavior of device II at different resistances (curves are offset vertically for clarity.)

The MR curves for all of the samples in the metallic regimes, with resistances below $h / e^{2}$, do not exhibit abrupt transitions between the $\mathrm{P}$ and $\mathrm{AP}$ resistances when the magnetic field is swept but rather show more gradual behavior (Figure 3B, inset). The form of the MR curve also varies as a device's resistance is increased. This suggests that the position and structure of the domain wall in the constriction may change as the magnetic field is varied.

When the resistance of a device becomes greater than tens of kiloohms, the transport is dominated by electron tunneling. In this regime, most devices exhibit a clean switching behavior with well-defined P and AP states (Figure 2, inset). Even after the metal bridge is broken, the size of the tunneling gap can still be adjusted by further electromigration. The shape of the MR curve and the values of the switching fields do not change significantly as electromigration changes the tunnel gap, but the value of the MR and even its sign can fluctuate over a wide range (Figure 4). This suggests that the MR is sensitive to the details of the atomic structure near the tunnel gap. The tunneling current is flowing through just a few atoms on each of the electrodes, and the electronic structure at these atoms does not necessarily reflect the same degree of spin polarization as in the bulk of the ferromagnet. ${ }^{14}$ The histogram of MR values we measure over 38 values of resistance in 20 tunneling devices is shown in the inset of Figure 4. The MR values range from $-10 \%$ to a maximum of $85 \%$.

It is interesting to examine the bias voltage $(V)$ dependence of the conductance and the MR. In the low resistance regime 


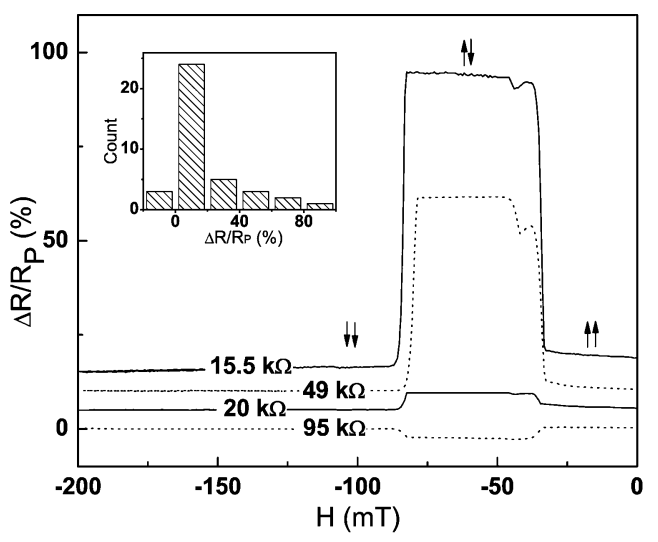

Figure 4. Evolution of magnetoresistance in the tunneling regime (device III) as the resistance of the tunneling gap is changed by electromigration (curves are vertically offset for clarity). Inset: distribution of magnetoresistances for devices in the tunneling regime $(>20 \mathrm{k} \Omega)$.

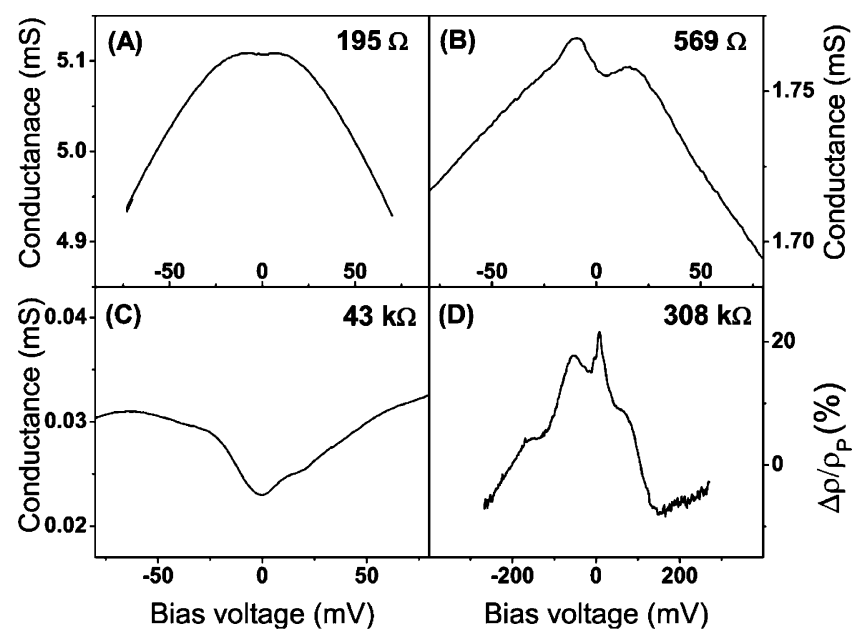

Figure 5. Differential conductance as a function of bias voltage for device IV at small (A), intermediate (B), and large (C) resistances. (D) Magnetoresistance as a function of bias for a tunneling device (device V).

( $<400 \Omega$ ), the MR is independent of $V$ and the conductance decreases with bias (Figure 5A), as it should because of increased backscattering at higher $V$ in metallic devices. For high-resistance tunneling devices, the conductance increases with $V$ (Figure 5C). In the intermediate regime (400 $\Omega$ to $25 \mathrm{k} \Omega$ ), the conductance typically increases with $V$ near $V$ $=0$ as in the tunneling regime but then decreases with $V$ at higher biases as in the metallic regime. We interpret this as the effect of having both metallic channels and tunneling channels contributing in parallel. The MR in the tunneling regime displays strong dependence on $V$ (Figure 5D). The exact form of the dependence differs from device to device, but typically the MR drops by a factor of 2 on the scale of $V=100 \mathrm{mV}$. This is similar to the behavior of standard magnetic tunnel junctions with oxide barriers ${ }^{13}$ and is in contrast to the STM experiments by Wulfhekel et al., ${ }^{26}$ in which no voltage dependence of the MR was found for a vacuum tunneling gap.

In summary, using a combination of electron beam lithography and controlled electromigration, we fabricate ferromagnetic junctions with tunable cross section, with sizes ranging from $100 \times 30 \mathrm{~nm}^{2}$ to near the atomic scale. Further electromigration opens a tunneling gap between the electrodes. These devices do not have any suspended parts and are stable against magnetostriction, magnetostatic, and thermal effects. We measure the low-temperature magnetoresistance as a function of the cross-section of the constriction. When the cross-section is larger than approximately 1 $\mathrm{nm}^{2}$ (corresponding to a resistance of $\sim 400 \Omega$ ) the MR is less than $3 \%$ and increases as the cross-section decreases, similar to the results of previous studies. As the contact diameter is decreased further, we observe first a minimum in the MR and sometimes a change in sign to small negative values, and then a strongly increasing positive MR as the contact approaches the atomic scale $(\sim 25 \mathrm{k} \Omega)$. For nearatomic-sized constrictions, we observe MR as high as $80 \%$, but find no devices in which the MR is as large as that reported previously for the ballistic magnetoresistance mechanism. ${ }^{5-7}$ In the tunneling regime, the MR values fluctuate over a wide range, $-10 \%$ to $85 \%$, even for small changes in the atomic structure near the constriction in a single device.

We note that Keane, Lu, and Natelson have posted independent results of a similar experiment recently. ${ }^{27}$

Acknowledgment. We thank O. Ozatay and A. Champagne for useful discussions and V. Sazonova for experimental help. This work was funded by the NSF (DMR0244713 and through the use of the Cornell NanoScale Science \& Technology Facility/NNIN) and by the ARO (DAAD19-01-1-0541).

\section{References}

(1) Kent, A. D.; Yu, J.; Rudiger, U.; Parkin, S. S. P. J. Phys.: Condens. Matter 2001, 13, R461.

(2) Bruno, P. Phys. Rev. Lett. 1999, 83, 2425.

(3) Levy, P. M.; Zhang, S. Phys. Rev. Lett. 1997, 79, 5110.

(4) Parkin, S. S. P. Annu. Rev. Mater. Sci. 1995, 25, 357.

(5) Garcia, N.; Muñoz, M.; Zhao, Y.-W. Phys. Rev. Lett. 1999, 82, 2923.

(6) Hua, S. Z.; Chopra, H. D. Phys. Rev. B 2003, 67, 060401.

(7) Chopra, H. D.; Sullivan, M. R.; Armstrong, J. N.; Hua, S. Z. Nat. Mater. 2005, 4, 832.

(8) Viret, M.; Berger, S.; Gabureac, M.; Ott, F.; Olligs, D.; Petej, I.; Gregg, J. F.; Fermon, C.; Francinet, G.; Le Goff, G. Phys. Rev. B 2002, 66, 220401(R).

(9) Gabureac, M.; Viret, M.; Ott, F.; Fermon, C. Phys. Rev. B 2004, 69, 100401.

(10) Yang, C.-S.; Zhang, C.; Redepenning, J.; Doudin, B. Appl. Phys. Lett. 2004, 84, 2865 .

(11) Montero, M. I.; Dumas, R. K.; Liu, G.; Viret, M.; Stoll, O. M.; Macedo, W. A. A.; Schuller, I. K. Phys. Rev. B 2004, 70, 184418.

(12) Egelhoff, W. F., Jr.; Gan, L.; Ettedgui, H.; Kadmon, Y.; Powell, C. J.; Chen, P. J.; Shapiro, A. J.; McMichael, R. D.; Mallet, J. J.; Moffat, T. P.; Stiles, M. D.; Svedberg, E. B. J. Magn. Magn. Mater. 2005, 287, 496.

(13) Tsymbal, E. Y.; Mryasov, O. N.; LeClair, P. R. J. Phys.: Condens. Matter 2003, 15, R109.

(14) Jacob, D.; Fernández-Rossier, J.; Palacios, J. J. Phys. Rev. B 2005 71, 220403(R)

(15) Ozatay, O.; Chalsani, P.; Emley, N. C.; Krivorotov, I. N.; Buhrman, R. A. J. Appl. Phys. 2004, 95, 7315.

(16) Pasupathy, A. N.; Bialczak, R. C.; Martinek, J.; Grose, J. E.; Donev, L. A. K.; McEuen, P. L.; Ralph, D. C. Science 2004, 306, 86.

(17) OOMMF is Object Oriented MicroMagnetic Framework, a micromagnetic simulation code available free from NIST at http:// math.nist.gov/oommf/.

(18) Guillorn, M. A.; Carr, D. W.; Tiberio, R. C.; Greenbaum, E.; Simpson, M. L. J. Vac. Sci. Technol., B 2000, 18, 1177. 
(19) Strachan, D. R.; Smith, D. E.; Johnston, D. E.; Park, T.-H.; Therien, M. J.; Bonnell, D. A.; Johnson, A. T. Appl. Phys. Lett. 2005, 86, 043109.

(20) Sordan, R.; Balasubramanian, K.; Burghard, B.; Kern, K. Appl. Phys. Lett. 2005, 87, 013106.

(21) Houck, A. A.; Labaziewicz, J.; Chan, E. K.; Folk, J. A.; Chuang, I. L. Nano Lett. 2005, 5, 1685 .

(22) Jansen, A. G. M.; van Gelder, A. P.; Wyder, P. J. Phys. C 1980, 13, 6073 .
(23) Lepadatu, S.; Xu, Y. B. Phys. Rev. Lett. 2004, 92, 127201.

(24) Agrait, N.; Yeyati, A. L.; van Ruitenbeek, J. M. Phys. Rep. 2003, $377,81$.

(25) Kim, T.-S. Phys. Rev. B 2005, 72, 024401.

(26) Wulfhekel, W.; Ding, H. F.; Kirschner, J. J. Magn. Magn. Mater 2002, 242-245, 47

(27) Keane, Z. K.; Yu, L. H.; Natelson, D. cond-mat/0510094.

NL0522936 